\title{
Removal of High Density Impulse Noise of Aerial Insulator Image
}

\author{
Cui Kebin ${ }^{1}$, Li Baoshu ${ }^{2}$, Yuan Jinsha ${ }^{3}$ and Wang Ping ${ }^{1}$ \\ ${ }^{1}$ Department of Computer, North China Electric Power University, Baoding, China, \\ 071003 \\ ${ }^{2}$ Department of Electrical Engineering, North China Electric Power University, \\ Baoding, China, 071003 \\ ${ }^{3}$ Department of Electronics, North China Electric Power University, Baoding, China, \\ 071003 \\ ncepuckb@163.com,libshu@263.net,ncepuyuanjs@163.com,pingwang@163.com
}

\begin{abstract}
Aiming at the impulse noise generated in capturing the images of insulator on power lines, a denoising method based on peer groups is proposed. The center pixel variance $\sigma_{\text {center }}$ is defined, the minimum of neighborhood variance and $\sigma_{\text {center }}$ is treated as threshold $\sigma_{\min }$ and the peer group is determined by comparing the relation between absolute value of gray value difference and $\sigma_{\min }$. According to the size of peer group and its complement set, center pixel is estimated when noisy pixels exist in the neighborhood window. Otherwise, the size of window is adjusted adaptively and center pixel is estimated on the basis of mean value of non-noisy pixels within adjusted window. The experimental results show that the method can get a higher peak signal to noise ratio, IEF and SSIM when there is high density impulse noise in an image.
\end{abstract}

Keywords: insulator; impulse noise; peer group; high density; denoising

\section{Introduction}

In recent years, using aerial images to determine the insulator failure has become an important way of transmission line inspection. However, impulse noise affects the image quality so seriously that to carry out the insulator fault diagnosis effectively, denoising algorithm must be used to remove noise and restore the true face of the image. Thus, how to choose good denoising algorithm becomes particularly important.

To remove the impulse noise in images, researchers all over the world have proposed many methods, which fall into two general categories. One is denoising in the frequency domain, in which wavelet denoising and hyper-wavelet denoising [3-5] act as representatives, the other is denoising in the spatial domain, including median filter, mean filter and denoising based on peer groups [6-14]. Median filter is the most widely used method by now, however, in the traditional median filter the gray value of the center of the image is substituted by the median value of its neighborhood, which will results in loss the most important details such as the edges and then blur the images. Especially when noise density is greater than $50 \%$, the defect becomes more obviously.

In addition, in literature [8], the authors proposed to achieve image enhancement by using Peer Group Averaging (PGA), which is used to realize image de-noising in literature [9-13]. When affected by noise pulses, the gray values of the image pixels will be either the maximum or the minimum value, and randomly distribute in the image. For 8-bit gray scale 
image, the gray values of 0 and 255 represent the pulse noise. In literature [8-13], all the pixels are processed rather than the 0 and 255 gray values which may be impulse noise. With the increase of the noisy pulse, the effect of the algorithm declines sharply. Thus, it is necessary to propose a de-noising algorithm aiming at the 0 and 255 gray values for highdensity impulse noise.

Based on analyzing the characteristic of high density noise, an adaptive de-noising algorithm based on modified peer groups is promoted in this paper, which is called MPGAD (Modified Peer Groups and Adaptive Denoising Algorithm). In this algorithm, only the pixels with gray value 0 or 255 are processed and even with high noise density, it shows good denoising performance.

\section{Peer Group}

A For a given pixel p, peer group is defined as a set of similar pixels in its neighborhood. When it comes to similarity, a variety of standards have been proposed by researchers. In literature [8], the absolute value of the difference between the gray value of the pixel and the center point is used to determine the peer group. Assuming $f(x, y)$ represents the gray value of $\mathrm{p}, f(s, t)$ represents the gray value of pixel $\mathrm{q}$ in its neighborhood, all the pixels match $|f(s, t)-f(x, y)| \leq m d$ consist of the peer group of $\mathrm{p}$. The principle of the method is simple, but different image has different characteristic, how to determine $m d$ reasonably is a problem. Several experiments must be done for each image to obtain better results, which is not suitable for the program to run automatically. In literature [9-14], peer group is determined by the sequence of the similarity between other pixel and the center point in the neighborhood. Assuming $\rho$ represents the similarity measurement between two pixels, $\left\{f_{1}, f_{2} \ldots, f_{n^{2}}\right\}$ represents all the pixels in $n \times n$ window, which we call $\mathrm{W}, f_{1}$ represents the center point pixel. The similarity measurement meets the following requirements:

$$
W=\left\{f_{(1)}, f_{(2)}, \ldots, f_{\left(n^{2}\right)}\right\}
$$

where, $f_{(i)}$ represents the ith pixel most similar to the center pixel。

$$
\rho\left(f_{1,} f_{(1)}\right) \geq \rho\left(f_{1}, f_{(2)}\right) \geq \ldots \rho\left(f_{1}, f_{\left(n^{2}\right)}\right)
$$

Obviously, $f_{(1)}=f_{1}$

The peer group of center pixel $f_{1}$, including m elements, is defined as:

$$
G_{m}^{f_{1}}=\left\{f_{(i)}, i=1, . ., m\right\}
$$

The selection of $\mathrm{m}$ is the key of this method. Fisher discriminant is firstly used in peer group to solve this problem in literature [9]. Assuming $\rho_{i}=\rho\left(f_{1}, f_{(i)}\right)=\left|f_{(i)}-f_{1}\right|$ represents the absolute value of gray value difference between $f_{(i)}$ and $f_{1}$, from the definition of $f_{(i)}$, we can get $\rho_{1} \leq \rho_{2} \leq \ldots \leq \rho_{n^{2}}$, thus, $\rho_{1}, \rho_{2}, \ldots, \rho_{n^{2}}$ is an increasing sequence of distances between pixels and the center point in the $n \times n$ neighborhood of $f_{1}$. Then classify $\rho_{i}$, $i=1, \ldots, n^{2}$ by Fisher discriminant to determine the number of elements in peer group of $f_{1}$, meanwhile, determine the pixels in it.

The objective function of Fisher discriminant is: 


$$
F(k)=\left|a_{1}-a_{2}\right|^{2} /\left(v_{1}+v_{2}\right), \quad \mathrm{k}=1,2, \ldots \mathrm{n}^{2}-1
$$

where,

$$
a_{1}=\sum_{i=1}^{k} \rho_{i} / k, \quad a_{2}=\sum_{i=k+1}^{n^{2}} \rho_{i} /\left(n^{2}-k+1\right), \quad v_{1}=\sum_{i=1}^{k}\left(\rho_{i}-a_{1}\right)^{2}, \quad v_{2}=\sum_{i=k+1}^{n^{2}}\left(\rho_{i}-a_{2}\right)^{2}
$$

The basic principle of this method is projection, which means projecting the highdimensional data to low dimensional space. The discriminant function is determined by the principle of maximum variance within class, minimum variance between classes. Therefore, calculating $F(k)$ to find the maximum and the corresponding value of $k$, which is used as the number of peer group Subsequently, find the corresponding pixels of the first k elements in the sequence of $\rho_{i}$ to compose the peer group of $f_{1}$.

It can be seen from the expression of Fisher discriminant that the number of peer group got by this method may not equal to $n^{2}$, which means that even if all the pixels in the neighborhood are similar to the center point, they will not be included in the same group.

\section{Adaptive Denoising Algorithm based on Modified Peer Groups- MPGAD}

\subsection{The Improvement of Peer Group Selection and the Adaptive Adjustment of Neighborhood Window}

In allusion to the shortage of Fisher discriminant that it is impossible for all the pixels in the neighborhood falling into the same peer group, center mean square error is defined based on MSE and a new method for selection of peer group based on them is promoted in this paper.

Peer group represents a collection of pixels similar to the center point and the MSE (mean square error) indicates the degree of dispersion between pixels. The smaller the MSE is, the smaller the degree of dispersion is and more similar between pixels. Therefore, it is analogy to the definition of peer group. The definition of MSE is:

$$
\sigma=\left(\sum_{k=1}^{3} \sum_{l=1}^{3}\left(x_{k l}-\bar{x}_{i j}\right)^{2} / n^{2}\right)^{1 / 2}
$$

MSE reflects the degree of dispersion between each point and the mean value, but peer group considers the degree of dispersion between each point and the center point. So CMSE (center mean square error) which represents the degree of dispersion between each point and the center point is defined as:

$$
\text { Definition 1: } \sigma_{\text {center }}=\left(\sum_{k=1}^{3} \sum_{l=1}^{3}\left(x_{k l}-x_{i j}\right)^{2} / n^{2}\right)^{1 / 2}
$$

Where $x_{i j}$ represents the gray value of the center point, $x_{k l}$ represents the gray value of the pixels in the $3 \times 3$ neighborhood $\mathrm{W}$ of the center point, $\bar{x}_{i j}$ represents the mean gray value of all the pixels in $\mathrm{W}$ and $\mathrm{n}$ is the size of $\mathrm{W}$.

CMSE $\sigma_{\text {center }}$ reflects the degree of dispersion between the center point and other pixels in the neighborhood. The larger CMSE is, the larger degree of dispersion between the pixel in neighborhood and the center point is, which means less similarity between them, whereas, the 
smaller CMSE is, the smaller degree of dispersion, the more similarity between the pixel and the center point.

To determine the peer group, not only the MSE $\sigma$ but also the CMSE $\sigma_{\text {center }}$ are

considered. Firstly, for all the pixels in the neighborhood $\left\{f_{1}, f_{2} \ldots, f_{n^{2}}\right\}$, determine the smaller one between $\sigma$ and $\sigma_{\text {center }}$, that is, $\sigma_{\min }=\min \left(\sigma, \sigma_{\text {center }}\right)$, secondly, calculate the absolute value $\mathrm{d}$ between the gray value of center point $f_{1}$ and other pixel $f_{i}$ in the neighborhood, and last determine whether $f_{i}$ is in peer group, when $d \leq \sigma_{\min }, f_{i}$ is the element of the peer group of $f_{1}$, or otherwise not. It is possible for all the pixels in the neighborhood falling into the same peer group in this method, which can avoid the shortage of Fisher discriminant.

For the circumstance all the pixels in the neighborhood are noisy (gray value is 0 or 255), a method to evaluate the gray value of the center point by adaptive adjustment of the neighborhood window is promoted. The method is described with following notations:

$N H_{n}(a, b)$ represents the set of the pixels in the $n \times n$ neighborhood of point $(a, b)$.

$N N_{n}(a, b)$ represents the set of the non-noisy pixels in the $n \times n$ neighborhood of point ( $a$, $b)$, whose mathematics form is:

$$
N N_{n}(a, b)=\left\{f_{i} \mid f_{i} \in N H_{n}(a, b) \wedge \operatorname{val}\left(f_{i}\right) \neq 0 \wedge \operatorname{val}\left(f_{i}\right) \neq 255\right\}
$$

$P G_{n}(a, b)$ stands for the peer group of point $(a, b)$ in its $n \times n$ neighborhood, whose mathematics form is:

$$
P G_{n}(a, b)=\left\{f_{i} \mid \operatorname{abs}\left(\operatorname{val}\left(f_{i}\right)-\operatorname{val}\left(f_{1}\right)\right) \leq \sigma_{\min }, f_{i} \in N H_{n}(a, b)\right\}
$$

$\overline{P G_{n}(a, b)}$ stands for the complementary set of $P G_{n \times n}(a, b)$ in the $n \times n$ neighborhood of point $(a, b)$, whose mathematics form is:

$$
\overline{P G_{n}(a, b)}=\left\{f_{i} \mid f_{i} \in N H_{n}(a, b) \wedge f_{i} \notin P G_{n}(a, b)\right\}
$$

$V^{N}$ represents the set of non-noisy pixels in set $\mathrm{V}$, the mathematic form is:

$$
V^{N}=\left\{f_{i} \mid f_{i} \in V \wedge \operatorname{val}\left(f_{i}\right) \neq 0 \wedge \operatorname{val}\left(f_{i}\right) \neq 255\right\}
$$

Where, $\operatorname{val}\left(f_{i}\right)$ stands for the gray value of pixel $f_{i}$

When the gray value of $(a, b)$ is 0 or 255 , firstly, determine $N N_{3}(a, b)$, if it is null, which means that all the pixels in the $3 \times 3$ neighborhood of $(a, b)$ is noise, then increase the size of neighborhood window with step of 2 , that is let $n=5$, Secondly, determine $N N_{5}(a, b)$, if it is null, increase the size of neighborhood window with step of 2 , repeat the process until $N N_{n}(a, b)$ is not null, then replace the gray value of $(a, b)$ by the mean gray value of all the pixels in $N N_{n}(a, b)$.

\subsection{Steps of the Algorithm}

For image $\mathrm{X}$, if the gray value of pixel $x(i, j)$ is 0 or 255 , it will be treated as impulse pixel. After processing the impulse pixel, the gray value of it will change, which will influence the 
calculation of other pixels. To avoid the influence, $\mathrm{Y}$ is introduced to represent the image after processing. The basic idea of the algorithm is first to detect the impulse noise and get the pixels in its $3 \times 3$ neighborhood, and then process them according to the number of nonoisy pixels.

The detail steps of the algorithm are as follow:

Step 1: To traverse all the pixels of the image one by one, if the gray value of the pixel is not equal to 0 or 255 , let $Y(i, j)=X(i, j)$ and continue on to the next pixel, else it is recognized as noise and go to step 2 .

Step 2: Let it be the center point, calculate set $V=N N_{3}(i, j)$ and get the number of elements of $\mathrm{V}$, denoted by NV. If $N_{V}>0$, turn to step 4 , else to step 3 .

Step 3: Let $n=n+2$, get set $V_{a}=N N_{n}(i, j)$, if the number of elements of set $V_{a}$ still equals to 0 , then repeat increasing the value of $\mathrm{n}$ with step of 2 until $V_{a}$ is not null. Replace the gray value of the center point by the mean gray value of all the pixels in $V_{a}$.

Step 4:Divide the pixels in $3 \times 3$ neighborhood of the center point into two groups $V_{1}$ and $V_{2}, V_{1}=P G_{3}(a, b), \quad V_{2}=\overline{P G_{n}(a, b)}$, remove the impulse noise from $V_{1}$ and $V_{2}$, we can get $V_{1}^{N}$ and $V_{2}^{N}$, compare the number of elements of $V_{1}^{N}$ and $V_{2}^{N}$, the mean gray value of all the pixels in the one with more elements is used to replace the gray value of the center point.

Step 5: Output image $\mathrm{X}$ and $\mathrm{Y}$.

\section{Experiments and Results}

To show the superiority of proposed algorithm, PSNR、SSIM and IEF are selected to evaluate the performance of it and compare it with several good algorithms. The definition of PSNR、SSIM and IEF are defined in (10) (11) and (12), respectively.

$$
P S N R=10 \log _{10}\left(\frac{255^{2}}{M S E}\right)
$$

where, $M S E=\frac{\sum_{i} \sum_{j}\left(x_{i j}-y_{i j}\right)^{2}}{M N}$

PSNR represents the Peak Signal to Noise Ratio, the larger the better.

$$
I E F=\frac{\left(\sum_{i} \sum_{j}\left(r_{i j}-x_{i j}\right)^{2}\right.}{\left(\sum_{i} \sum_{j} y_{i j}-x_{i j}\right)^{2}}
$$

IEF represents the Image Enhancement Factor, which is used to evaluate the edge reserving ability. The larger it is, the more edge information can be reserved.

$$
\operatorname{SSIM}(x, y)=\frac{\left(2 \mu_{x} \mu_{y}+C_{1}\right)\left(2 \sigma_{x y}+C_{2}\right)}{\left(\mu_{x}^{2}+\mu_{y}^{2}+C_{1}\right)\left(\sigma_{x}^{2}+\sigma_{y}^{2}+C_{2}\right)}
$$

where, $\quad \mu_{x}=\frac{1}{M N} \sum_{i} \sum_{j} x_{i j}, \mu_{y}=\frac{1}{M N} \sum_{i} \sum_{j} y_{i j}, \sigma_{x}=\left(\frac{1}{M N-1} \sum_{i} \sum_{j}\left(x_{i j}-\mu_{x}\right)^{2}\right)^{\frac{1}{2}}$, 


$$
\sigma_{y}=\left(\frac{1}{M N-1} \sum_{i} \sum_{j}\left(y_{i j}-\mu_{y}\right)^{2}\right)^{\frac{1}{2}}, \sigma_{x y}=\left(\frac{1}{M N-1} \sum_{i} \sum_{j}\left(x_{i j}-\mu_{x}\right)\left(y_{i j}-\mu_{y}\right)\right.
$$

$C 1$ and $C 2$ are adjusting parameters and here $C 1=0.01$ and $C 2=0.03$.

SSIM represents the structural similarity, which is used to measure the similarity of two images, the larger the better and the maximum is 1. Compare with the traditional measurement of image quality, such as PSNR, SSIM more accords with human visual judgment.

In (10) (11) (12), $x$ represents the original image, $y$ represents the denoising image, $r$ represents the corrupted image and $M N$ represents the size of image.

Several good algorithms, including MF, DBAIN in literature [15] and NAFSM in literature [16], have a good performance for denoising. All these algorithms are tested with standard image lean.jpg and an aerial insulator image insulator.jpg the size of which are $512 \times 512$ and $1037 \times 345$, respectively. A wide range of noise density varied from $10 \%$ to $90 \%$ with increments of $10 \%$ and an extremely high one $95 \%$ will be tested. Parameters should be set in NAFSM, here $\mathrm{T} 1=10$ and $\mathrm{T} 2=30$. Because the randomness of the location of corrupted noisy pixels, even with the same image, same noisy density, different results will be given by twice tests, especially the value of IEF. To reflect the performance of different algorithm, ten tests will be done with the same image and same noisy density, results of ten tests will be averaged as the final result.

Table 1. Table Comparing PSNR Values of Different Algorithms for lena at Different Noise Densities

\begin{tabular}{ccccc}
\hline Noise & MF & DBAIN & NAFSM & MPGAD \\
\hline $10 \%$ & 33.27 & 41.48 & 38.77 & 42.16 \\
$20 \%$ & 29.21 & 37.48 & 35.61 & 38.48 \\
$30 \%$ & 23.60 & 34.75 & 33.66 & 36.27 \\
$40 \%$ & 18.86 & 32.39 & 32.22 & 34.56 \\
$50 \%$ & 15.24 & 30.17 & 31.03 & 32.97 \\
$60 \%$ & 12.40 & 27.96 & 29.88 & 31.52 \\
$70 \%$ & 9.96 & 25.64 & 28.62 & 30.12 \\
$80 \%$ & 8.14 & 23.1 & 27.06 & 28.46 \\
$90 \%$ & 6.67 & 19.75 & 23.57 & 26.21 \\
$95 \%$ & 5.99 & 17.15 & 17.02 & 23.97 \\
\hline
\end{tabular}

Table 2. Table Comparing SSIM Values of Different Algorithms for lena at Different Noise Densities

\begin{tabular}{ccccc}
\hline Noise & MF & DBAIN & NAFSM & MPGAD \\
\hline $10 \%$ & 0.9441 & 0.9886 & 0.9836 & 0.9935 \\
$20 \%$ & 0.8951 & 0.9739 & 0.9664 & 0.9855 \\
$30 \%$ & 0.7399 & 0.9555 & 0.9478 & 0.9760 \\
$40 \%$ & 0.4738 & 0.9309 & 0.9278 & 0.9647 \\
$50 \%$ & 0.2400 & 0.8982 & 0.9061 & 0.9502 \\
$60 \%$ & 0.1084 & 0.8538 & 0.8806 & 0.9314 \\
$70 \%$ & 0.0484 & 0.7937 & 0.8482 & 0.9056 \\
$80 \%$ & 0.0240 & 0.7089 & 0.8009 & 0.8687 \\
$90 \%$ & 0.0127 & 0.5792 & 0.6828 & 0.8005 \\
$95 \%$ & 0.0071 & 0.4739 & 0.4027 & 0.7259 \\
\hline
\end{tabular}


Table 3. Table Comparing IEF Values of Different Algorithms for lena at Different Noise Densities

\begin{tabular}{ccccc}
\hline Noise & MF & DBAIN & NAFSM & MPGAD \\
\hline $10 \%$ & 60.80 & 402.24 & 215.01 & 467.15 \\
$20 \%$ & 47.39 & 319.86 & 207.39 & 400.42 \\
$30 \%$ & 19.71 & 255.46 & 198.9 & 362.92 \\
$40 \%$ & 8.82 & 197.86 & 190.37 & 326.78 \\
$50 \%$ & 4.76 & 148.3 & 181.01 & 283.19 \\
$60 \%$ & 2.97 & 107.2 & 166.7 & 243.31 \\
$70 \%$ & 1.99 & 73.18 & 145.36 & 204.39 \\
$80 \%$ & 1.49 & 46.68 & 116.27 & 160.60 \\
$90 \%$ & 1.19 & 24.23 & 58.43 & 107.22 \\
$95 \%$ & 1.08 & 14.08 & 13.65 & 67.79 \\
\hline
\end{tabular}

Table 4. Table Comparing PSNR Values of Different Algorithms for Insulator at Different Noise Densities

\begin{tabular}{ccccc}
\hline Noise & MF & DBAIN & NAFSM & MPGAD \\
\hline $10 \%$ & 36.35 & 45.49 & 42.91 & 45.26 \\
$20 \%$ & 30.07 & 40.72 & 39.22 & 41.15 \\
$30 \%$ & 24.16 & 37.64 & 37.18 & 38.58 \\
$40 \%$ & 19.77 & 34.99 & 35.48 & 36.78 \\
$50 \%$ & 15.56 & 32.67 & 34.03 & 35.15 \\
$60 \%$ & 12.49 & 30.32 & 32.67 & 33.69 \\
$70 \%$ & 10.21 & 27.99 & 31.15 & 32.64 \\
$80 \%$ & 8.26 & 25.67 & 29.34 & 30.89 \\
$90 \%$ & 6.77 & 22.77 & 25.05 & 28.68 \\
$95 \%$ & 6.15 & 20.51 & 17.52 & 26.49 \\
\hline
\end{tabular}

Table 5. Table Comparing SSIM Values of Different Algorithms for Insulator at Different Noise Densities

\begin{tabular}{ccccc}
\hline Noise & MF & DBAIN & NAFSM & MPGAD \\
\hline $10 \%$ & 0.9782 & 0.9971 & 0.9955 & 0.9972 \\
$20 \%$ & 0.9273 & 0.9926 & 0.9902 & 0.9934 \\
$30 \%$ & 0.7417 & 0.9845 & 0.9847 & 0.9888 \\
$40 \%$ & 0.4367 & 0.9776 & 0.9784 & 0.9836 \\
$50 \%$ & 0.1906 & 0.9655 & 0.9710 & 0.9771 \\
$60 \%$ & 0.0751 & 0.9487 & 0.9615 & 0.9690 \\
$70 \%$ & 0.0357 & 0.9250 & 0.9475 & 0.9587 \\
$80 \%$ & 0.0178 & 0.8922 & 0.9209 & 0.9449 \\
$90 \%$ & 0.0094 & 0.8403 & 0.8109 & 0.9192 \\
$95 \%$ & 0.0062 & 0.8022 & 0.4611 & 0.8905 \\
\hline
\end{tabular}




\section{Table 6. Table Comparing IEF Values of Different Algorithms for Insulator at Different Noise Densities}

\begin{tabular}{ccccc}
\hline Noise & MF & DBAIN & NAFSM & MPGAD \\
\hline $10 \%$ & 119.54 & 973.57 & 538.3 & 905.90 \\
$20 \%$ & 56.24 & 651.65 & 461.68 & 743.66 \\
$30 \%$ & 21.57 & 481.31 & 432.51 & 595.83 \\
$40 \%$ & 9.33 & 349.44 & 389.97 & 525.80 \\
$50 \%$ & 4.95 & 255.33 & 350.72 & 451.20 \\
$60 \%$ & 2.94 & 178.12 & 305.98 & 382.72 \\
$70 \%$ & 2.02 & 122.49 & 253.21 & 328.22 \\
$80 \%$ & 1.48 & 81.55 & 189.70 & 269.97 \\
$90 \%$ & 1.18 & 46.66 & 79.15 & 183.25 \\
$95 \%$ & 1.09 & 29.81 & 14.90 & 116.76 \\
\hline
\end{tabular}

The comparison of PSNR, SSIM and IEF values of different algorithms at different noise densities for lena and insulator is showed in Table 1 to Table 6, respectively.

From Table 1 to Table 6, it is observed that the performance of the proposed algorithm is better than the existing algorithms for both the informative standard image lena and insulator with clear target image, especially on the measurement SSIM, the proposed algorithm has obvious advantage. Even at $95 \%$, the values of PSNR, SSIM and IEF still maintain at a high level, which means the proposed algorithm get better restoration of the original image.

Plots of PSNR, SSIM and IEF against noise densities for lean are shown in Figure 1, Figure 2 and Figure 3, repetitively. It can be clearly seen from the figures that the proposed algorithm has excellent denoising ability when the density is over $50 \%$. Figure 4 is the original image of aerial insulator.

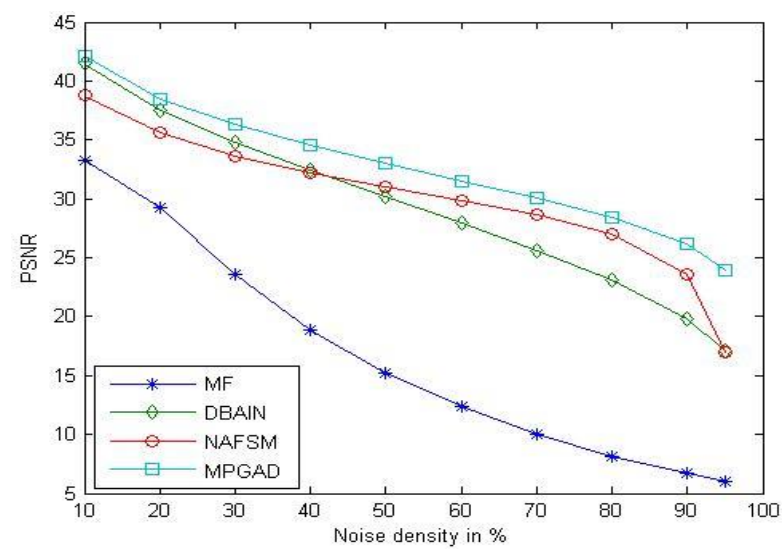

Figure 1. Results in PSNR for lena at Various Noise Levels for Different Algorithms 


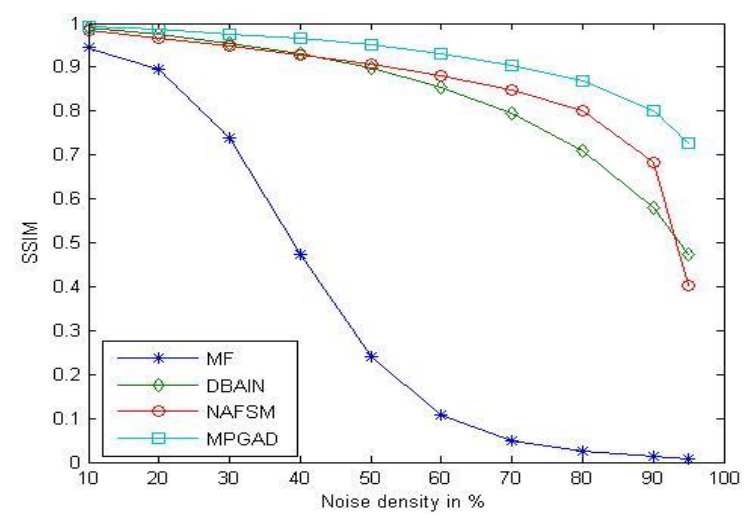

Figure 2. Results in SSIM for Lena at Various Noise Levels for Different Algorithms

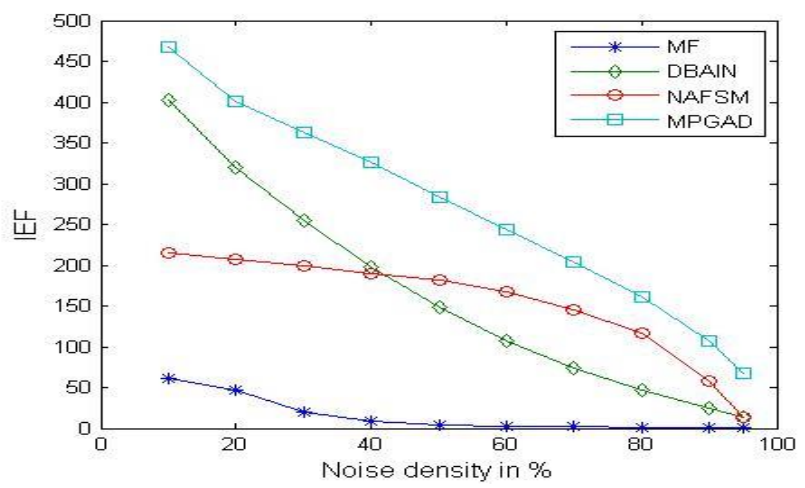

Figure 3. Results in IEF for lena at Various Noise Levels for Different Algorithms

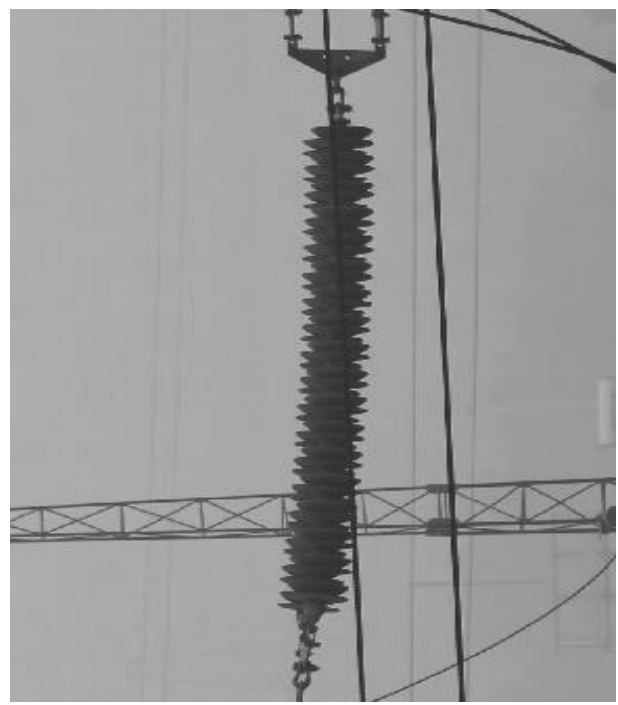

Figure 4. The Original Image of Aerial Insulator 
Figure 5 shows the outputs of different algorithms for lena and insulator at noise density $80 \%$ and $95 \%$.

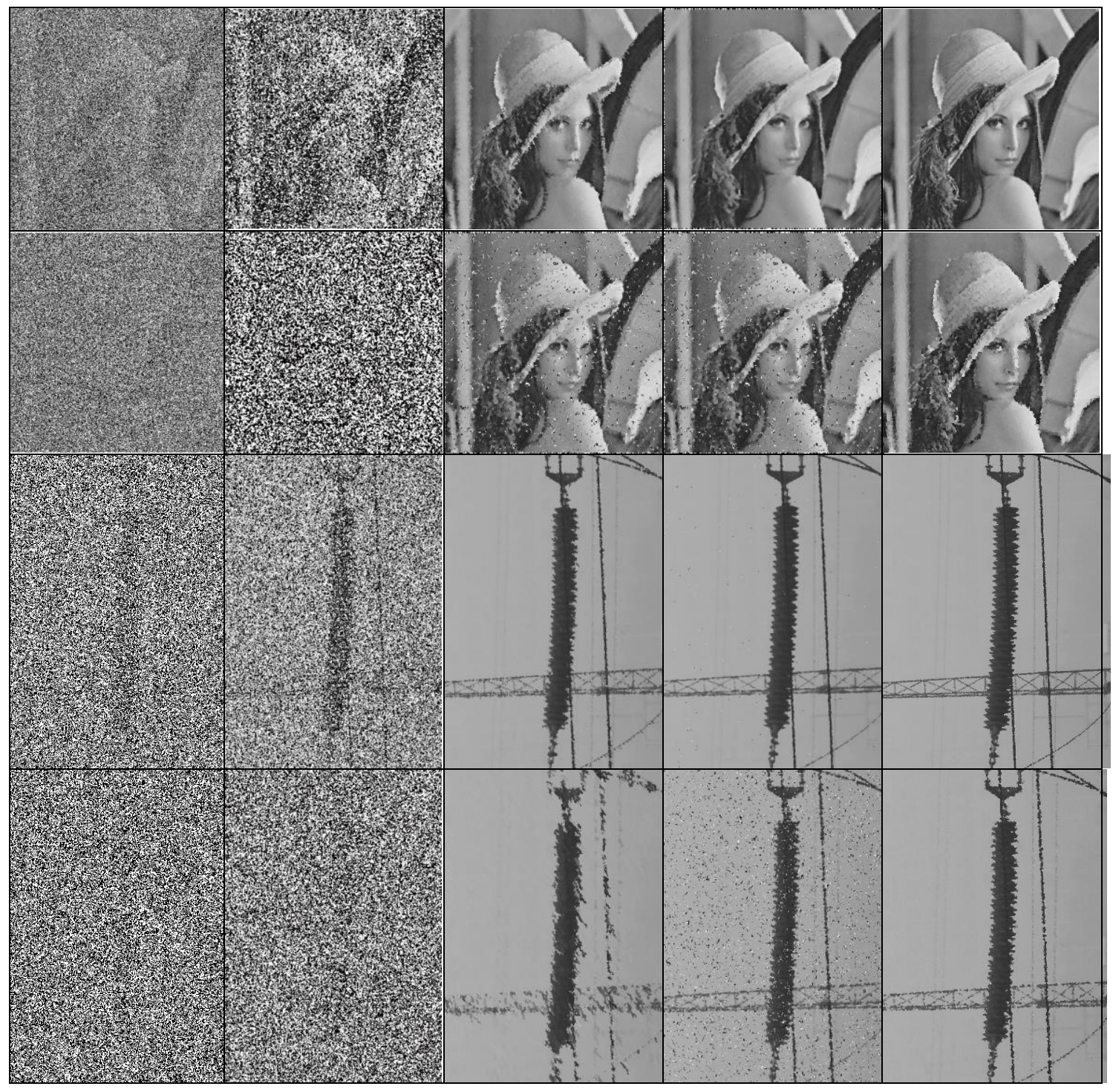

Figure 5. Restoration Results of Different Filters for lena and Insulator at Noisy Density $80 \%$ and $95 \%$

\section{Conclusion}

In allusion to the rapid declines in denoising ability of existing algorithms, a new denoising algorithm based on modified peer group is proposed and is used for aerial insulator image. Experiments have been done to test the algorithm by different measurements. Simulation results show that our algorithm performs much better than several standing algorithms existing, especially when the noise density is over $50 \%$. Even at a very high noise density $90 \%$ or $95 \%$, the details and edges are reserved by our algorithm and values of PSNR, SSIM and IEF are still satisfactory, which shows that the proposed algorithm is efficient for removal of high-density noise. 


\section{Acknowledgements}

This study was supported by "the Fundamental Research Funds for the Central Universities (N0. 2014MS134)."

\section{References}

[1] Z. S. Li, W. L. Li, J. G. Yao and Y. J. Yang, "On-site Detection of Pollution Level of Insulators Based on Infrared-thermal-image Processing", Proceedings of the CSEE, vol. 30, no. 4, (2010), pp. 132-138.

[2] F. J. Sun, Z. H. Yang, Y. Y. Li and J. Q. Yuan, "Methods of transmission line target recognition", Journal of Image and Graphics, vol. 17, no. 3, (2012), pp. 349-356.

[3] A. Pizurica, W. Philips, I. Lemahieu and M. Acheroy, "A versatile wavelet domain noise filteration technique for medical imaging”, IEEE Trans on Med Imaging, vol. 22, no. 3, (2003), pp. 323-331.

[4] F. Luisier, T. Blu and M. Unser, "A new SURE approach to image denoising: interscale orthonormal wavelet thresholding”, IEEE Trans Image Process, vol. 16, no. 3, (2007), pp. 593-606.

[5] J. Sun and Y. Feng, "An Improved Contourlet Transform and its Application in Image Denosing”, Journal of Computer-Aided Design \& Computer Graphics, vol. 21, no. 11, (2009), pp. 1612-1617.

[6] X. Zhang, b. Xu and S. Dong, "Adaptive Median Filtering for Image Processing", Journal of ComputerAided Design \& Computer Graphics, vol. 17, no. 2, (2005), pp. 295-299.

[7] J. Shan, "Non-local means denoising algorithm with enhanced weight function", Journal of Image and Graphics, vol. 17, no. 10, (2012), pp. 1227-1231.

[8] C. Kenney, Y. Deng and B. S. Manjunath, et al. "Peer group image enhancement", IEEE Trans Image Process, vol. 10, no. 2, (2001), pp. 326-334.

[9] Y. Deng, C. Kenney, M. S. Moore and B. S. Manjunath, "Peer group filtering and perceptual color image quantization", IEEE International Symposium on Circuits and Systems, vol. 4, (1999), pp. 21-24.

[10] H. He, Y. Qian and L. Wang, et al. "Insulator Image Denoising Based on Pixei Peer Groups and Neighbor Groups", Computer Science, vol. 39, no. 6, (2012), pp. 283-284, 307.

[11] S. Morillas, V. Gregori and A. Hervás, "Fuzzy peer groups for reducing mixed Gaussian-impulse noise from color images", IEEE Transactions on Image Processing, vol. 18, no. 7, (2009), pp. 1452-1466.

[12] Y. Deng, C. Kenney and M. S. Moore, et al. "Peer group filtering and perceptual color image quantization [C]", IEEE International Symposium on Circuits and Systems, (1999) May 30- Jun 02, Orlando, America.

[13] G. Hewer, C. Kenney, L. Peterson, A. van Nevel, "Applied partial differential variational techniques", Proceedings of International Conference on Image Processing, (1997) October 26-29, Santa Barbara, America.

[14] J. Y. F. Ho, "Peer region determination based impulsive noise detection", 2003 IEEE International Conference on Acoustics, Speech, and Signal Processing, (2003) April 6-10, Hong Kong, China.

[15] K. S. Srinivasan and D. Ebenezer, „A new fast and efficient decision-based algorithm for removal of highdensity impulse noise”, IEEE Signal Processing Letters, vol. 14, no 3, (2007), pp. 189-192.

[16] K. V. T. Kenny, A. M. Nor Isa, "Noise adaptive fuzzy switching median filter for salt and pepper noise reduction", IEEE Signal Processing Letters, vol. 17, no. 3, (2010), pp. 281-284.

[17] Z. Wang, A. C. Bovik, H. R. Sheikh and E.P. Simoncelli, "Image quality assessment: From error visibility to structural similarity [J]”, IEEE Transaction on Image Processing, vol. 13, no. 4, (2004), pp. 600-612.

\section{Authors}

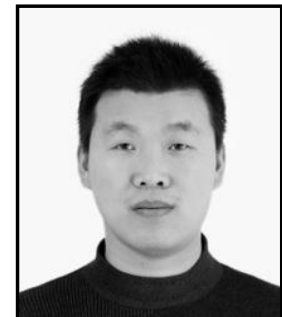

Kebin Cui, he is currently pursuing the Ph.D. degree from the Department of Electrical Engineering, North China Electric Power University, Baoding, China. He is currently a Lecturer with the Department of Computer, North China Electric Power University, His research interests are the application of image processing in electric power system, including image denoising, image segmentation, feature extraction, image fusion and so on. 


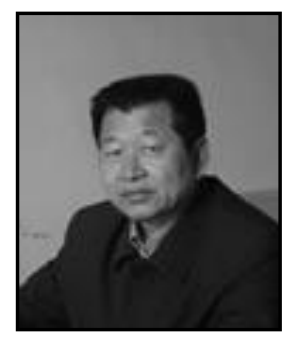

Baoshu Li, he is currently a Professor with the Department of Electrical Engineering, North China Electric Power University, Baoding China. His research interests are in the areas of image processing technology in power system applications, technology and power of modern electromagnetic measuring and Electrical equipment condition monitoring.

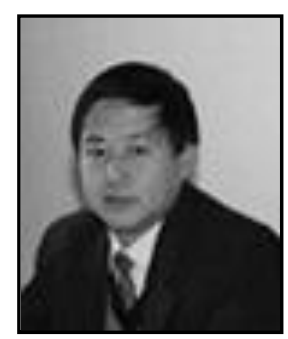

Jinsha Yuan, he is currently a Professor with the Department of Electronic, North China Electric Power University, Baoding China. His research interests are in the areas of image processing technology in power system applications, Computer information processing and Electromagnetic Calculation

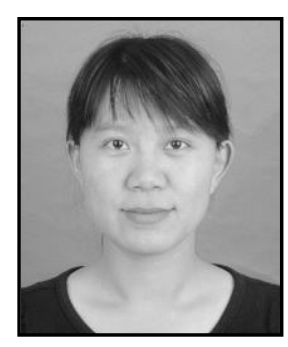

Ping Wang, she is currently a Lecturer with the Department of Computer, North China Electric Power University, Baoding China. Her research interests are in the areas of image denoising, image segmentation and Artificial intelligence. 